\title{
Modeling incompressible flows at low and high Reynolds numbers via a finite calculus-finite element approach
}

\author{
Eugenio Oñate *, Aleix Valls, Julio García \\ International Center for Numerical Methods in Engineering $(C I M N E)^{l}$, Universidad Politecnica de Cataluña, 08034 Barcelona, Spain
}

\begin{abstract}
We present a formulation for incompressible flows analysis using the finite element method (FEM). The necessary stabilization for dealing with convective effects and the incompressibility condition is modeled via the finite calculus (FIC) method. The stabilization terms introduced by the FIC formulation allow to solve a wide range of fluid flow problems for low and high Reynolds numbers flows without the need for a turbulence model. Examples of application of the FIC/FEM formulation to the analysis of 2D and 3D incompressible flows with moderate and large Reynolds numbers are presented.
\end{abstract}

Keywords: Incompressible flows; High Reynolds numbers; Finite calculus; Finite element method

\section{Introduction}

Many problems in mechanics are characterized by the existence of numerical values of the geometrical and/ or physical parameters differing in several orders of magnitude. Typical examples are convection-diffusionabsorption problems where high values of the convection or the absorption terms lead to sharp boundary and/or internal layers along which the numerical solution can change in many orders of magnitude. A similar problem is found in the analysis of thin discontinuity layers in solids, such as in the case of fracture in concrete, rock or geomaterials, or in shock waves in compressible fluids. A traditional multiscale problem is that of a turbulent fluid, where high gradients of the velocity field occur along random directions of the flow. The transition from a compressible to an incompressible solid or fluid can also be considered as a multiscale problem, in the sense that the propagation speed of sound changes from a finite value (for the compressible case) to an infinite value (in the case of incompressibility). Indeed, problems where many different scales of the material

\footnotetext{
* Corresponding author. Tel.: +34 932057016; fax: +34 934016517.

E-mail address: onate@cimne.upc.edu (E. Oñate).

${ }^{1}$ http://www.cimne.com.
} 
properties co-exist in a solid (such as in composites) or in a fluid are also classical examples of multiscale situations in mechanics.

It is well known that standard numerical methods such as the central finite difference (FD) method, the Galerkin finite element (FE) method and the finite volume (FV) method, among others, lead to unstable numerical solutions when applied to problems involving different scales, multiple constraints and/or high gradients. Examples of these situations are typical in the solution of convection-diffusion problems and incompressible flow problems in fluid mechanics using the standard Galerkin FE method or the central scheme in FD and FV methods [1,2,24].

The sources of the numerical instabilities in FE, FD and FV methods when solving above problems have been sought in the apparent unability of the Galerkin FE method and the analogous central difference scheme in FD and FV methods, to provide a numerical procedure able to capture the different scales appearing in the solution for all ranges of the physical parameters [1,2]. Typical examples are the spurious numerical oscillations in convection-diffusion problems for high values of the convective terms. The same type of oscillations are found in turbulent flows and in regions next to sharp internal layers appearing in high speed compressible flows (shocks). A similar problem of different nature emerges in the solution of incompressible problems in fluid and solid mechanics. Here the difficulties in satisfying the incompressibility constraint limit the choices of the approximation for the velocity (or displacement) variables and the pressure $[1,24]$.

Much effort has been spent in the context of the finite element method (FEM) in developing the so called stabilized numerical methods overcoming the two main sources of instability in incompressible flow analysis, namely those originated by the high values of the convective terms and those induced by the difficulty in satisfying the incompressibility condition.

The first attempts to correct the underdiffusive character of the Galerkin FEM for high convection flows were
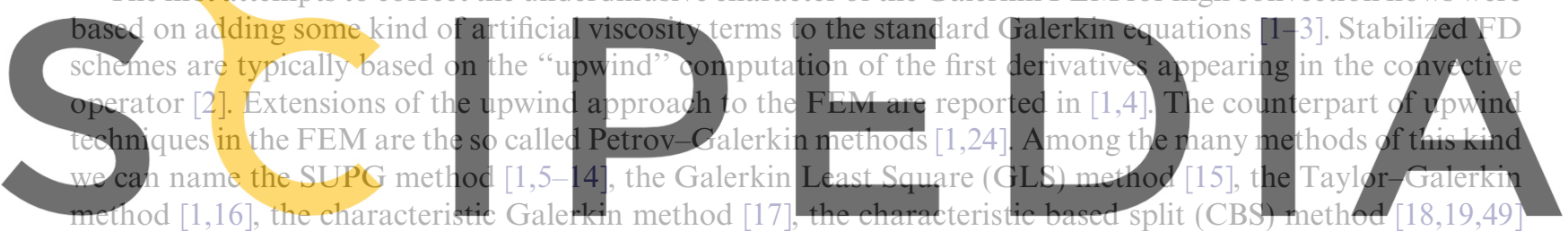

and the subgrid scale (SS) method [20-23]. A review of some of these methods can be found in [24].

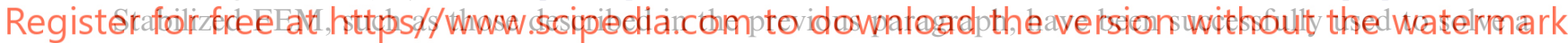
wide range of incompressible fluid mechanics problems. The intrinsic dissipative properties of the stabilization terms (which can interpreted as an additional viscosity) typically suffices to yield good results for low and moderate values of the Reynolds number $(R e)$. For high values of $R e$ most stabilized FEM fail to provide physically meaningful results and the numerical solution is often unstable or inaccurate. The introduction of a turbulence model is mandatory in order to obtain meaningful results in these cases.

Recent attempts to develop a unified relativity theory in physics adequate for dealing with the whole spectrum of scales in the universe, from the Plank atomistic scale $\left(10^{-33} \mathrm{~cm}\right)$ to the cosmological scale $\left(10^{+28} \mathrm{~cm}\right)$ indicate that a suitable mathematical model should incorporate the effect of the different scales within the governing equations of the model [25]. Failing to include these scale terms may lead to unstable behaviour of the equations when solved numerically for very different values of the physical, geometrical and/or time parameters of the problem.

The finite calculus (FIC) method developed by Oñate and co-workers [26-49] is a consistent procedure to re-formulate the governing equation in mechanics introducing new terms involving characteristic space and time dimensions into the equations. The modified equations are derived by invoking the balance laws in mechanics in a space-time domain of finite size. The new terms introduced by the FIC approach are essential to obtain physical (stable) numerical solutions for all ranges of the parameters governing the physical problem.

The merit of the modified equations via the FIC approach is that they lead to stabilized schemes using any numerical method. In addition, the different stabilized FD, FE and FV methods typically used in practice can be recovered using the FIC equations [26,27].

The FIC/FEM formulation has proven to be very effective for the solution of a wide class of problems, such as convection-diffusion [26-33] and convection-diffusion-reaction [34-36] with arbitrary high gradients, 
incompressible flow problems accounting for free surface effects and fluid-structure interaction situations [37$46]$ and quasi and fully incompressible problems in solid mechanics [47-49].

This paper extends the work recently presented in $[45,46]$ where an enhanced stabilized FEM for incompressible flows was derived via FIC. The FIC approach introduces additional terms in the classical differential equations of momentum and mass balance of infinitesimal fluid mechanics. The FIC terms have a matrix form and are a function of characteristic length dimensions related to the finite element sizes and also to the form of the numerical solution. A procedure to compute the characteristic length parameters is described. The FIC terms in the modified governing equations provide the necessary stabilization to the discrete equations obtained via the standard Galerkin FEM. The resulting FIC/FEM formulation allows to use low order finite elements (such as linear triangles and tetrahedra) with an equal order approximation for the velocity and the pressure variables.

It is shown in this paper that the non linear stabilization terms introduced by the FIC/FEM formulation can be used to solve accurately high Re number flows without the need of introducing any turbulence model. The FIC/FEM formulation described here therefore provides a straightforward procedure for solving a wide class of flow problems from low to high Reynolds numbers. The good results obtained in the $2 \mathrm{D}$ and $3 \mathrm{D}$ examples presented open the door for the reinterpretation of the (nonlinear) FIC stabilization terms as a turbulent model. The advantage of this analogy is that the FIC terms are derived from basic principles, such as balance of momentum and mass and, in conjunction with a numerical method such as the FEM, they provide a straightforward procedure for the analysis of complex problems in fluid mechanics.

The layout of the paper is the following. In the next section the FIC equations for incompressible flows with matrix stabilization terms are presented. The finite element discretization is introduced and the resulting matrix equations are given. A fractional step scheme for the transient solution is detailed. Examples of appli-
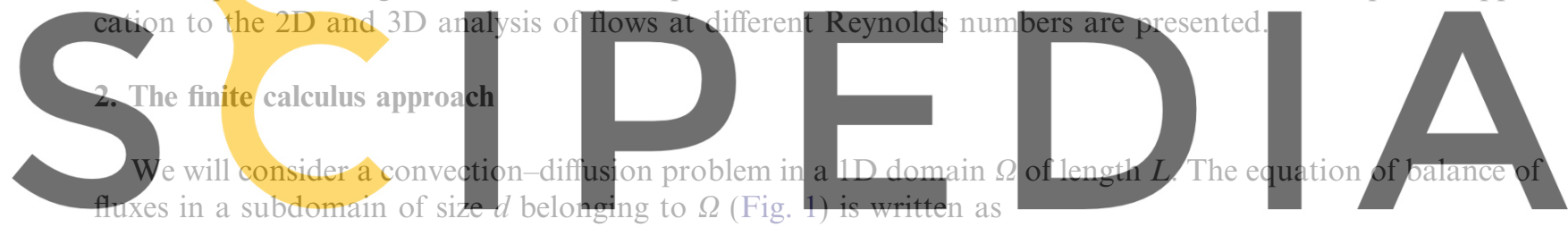

Register for free at https//www.scipedia.com to download the version without the watermark where $q_{A}$ and $q_{B}$ are the incoming and outgoing fluxes at points $A$ and $B$, respectively. The flux $q$ includes both convective and diffusive terms; i.e. $q=u \phi-k \frac{\mathrm{d} \phi}{\mathrm{d} x}$, where $\phi$ is the transported variable (i.e. the temperature in a thermal problem), $u$ is the velocity and $k$ is the diffusivity of the material. For simplicity the density and the specific heat constant have been assumed to have a unit value.

Let us express now the fluxes $q_{A}$ and $q_{B}$ in terms of the flux at an arbitrary point $C$ within the balance domain (Fig. 1). Expanding $q_{A}$ and $q_{B}$ in Taylor series around point $C$ up to second order terms gives

$$
q_{A}=q_{C}-\left.d_{1} \frac{\mathrm{d} q}{\mathrm{~d} x}\right|_{C}+\left.\frac{d_{1}^{2}}{2} \frac{\mathrm{d}^{2} q}{\mathrm{~d} x^{2}}\right|_{C}+\mathrm{O}\left(d_{1}^{3}\right), \quad q_{B}=q_{C}+\left.d_{2} \frac{\mathrm{d} q}{\mathrm{~d} x}\right|_{C}+\left.\frac{d_{2}^{2}}{2} \frac{\mathrm{d}^{2} q}{\mathrm{~d} x^{2}}\right|_{C}+\mathrm{O}\left(d_{2}^{3}\right)
$$

Substituting Eq. (2) into Eq. (1) gives after simplification

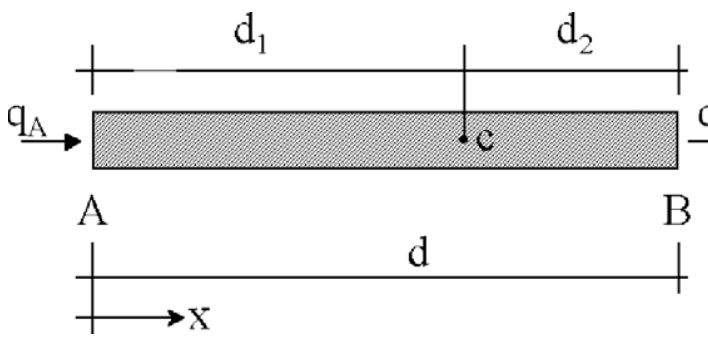

Fig. 1. Equilibrium of fluxes in a space balance domain of finite size. 


$$
\frac{\mathrm{d} q}{\mathrm{~d} x}-\frac{h}{2} \frac{\mathrm{d}^{2} q}{\mathrm{~d} x^{2}}=0
$$

where $h=d_{1}-d_{2}$ and all the derivatives are computed at the arbitrary point $C$.

Standard calculus theory assumes that the domain $d$ is of infinitesimal size and the resulting balance equation is simply $\frac{\mathrm{d} q}{\mathrm{~d} x}=0$. We will relax this assumption and allow the space balance domain to have a finite size. The new balance Eq. (3) incorporates now the underlined term which introduces the characteristic length $h$. Obviously, accounting for higher order terms in Eq. (2) would lead to new terms in Eq. (3) involving higher powers of $h$.

Distance $h$ in Eq. (3) can be interpreted as a free parameter depending on the location of point $C$ within the balance domain. Note that $-d \leqslant h \leqslant d$ and, hence, $h$ can take a negative value. At the discrete solution level the domain $d$ should be replaced by the balance domain around a node. This gives for an equal size discretization $-l^{\mathrm{e}} \leqslant h \leqslant l^{\mathrm{e}}$ where $l^{\mathrm{e}}$ is the element or cell dimension. The fact that Eq. (3) is the exact balance equation (up to second order terms) for any 1D domain of finite size and that the position of point $C$ is arbitrary, can be used to derive numerical schemes with enhanced properties simply by computing the characteristic length parameter from an adequate "optimality" rule, such as requiring to an smaller error in the numerical solution [26-36].

Consider, for instance, the 1D convection-diffusion problem. Neglecting third order derivatives of $\phi$, Eq. (3) can be rewritten in terms of $\phi$ as

$$
-u \frac{\mathrm{d} \phi}{\mathrm{d} x}+\left(k+\frac{u h}{2}\right) \frac{\mathrm{d}^{2} \phi}{\mathrm{d} x^{2}}=0
$$

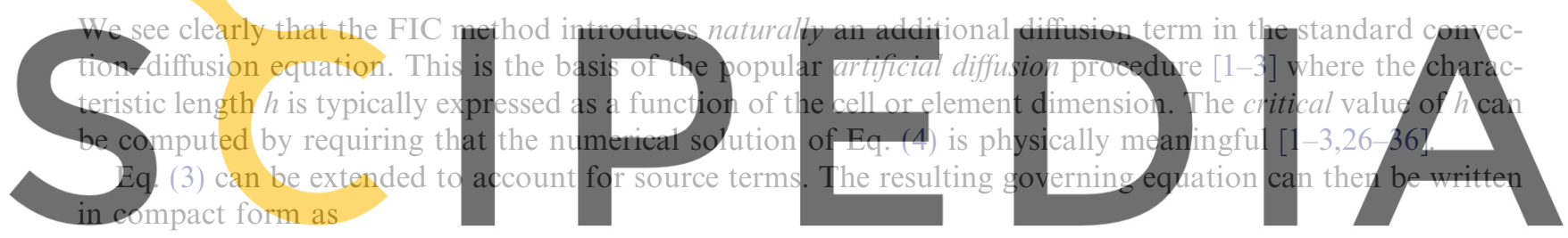

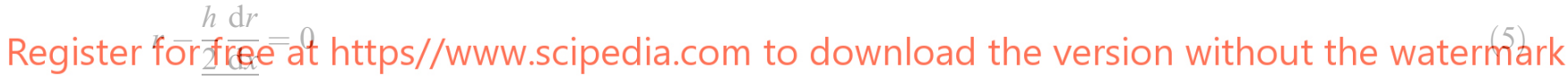

with

$$
r:=-u \frac{\mathrm{d} \phi}{\mathrm{d} x}+\frac{\mathrm{d}}{\mathrm{d} x}\left(k \frac{\mathrm{d} \phi}{\mathrm{d} x}\right)+Q
$$

where $Q$ is the external source. The essential (Dirichlet) boundary condition for Eq. (5) is $\phi=\bar{\phi}$ on $\Gamma_{\phi}$ where $\Gamma_{\phi}$ is the boundary where the prescribed value $\bar{\phi}$ is imposed. For consistency a stabilized Neumann boundary condition must be obtained as described next.

Note that for $h=0$ the classical balance equation of the infinitesimal theory $(r=0)$ is obtained.

Let us consider a balance domain next to a Neumann boundary point $B$ (Fig. 2)). The length of the balance segment $A B$ next to the boundary is taken as one half of the characteristic length $h$ for the interior domain. The balance equation, assuming a constant distribution for the source $Q$, is

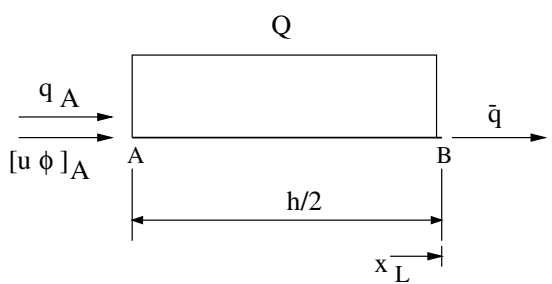

Fig. 2. Balance domain next to a Neumann boundary point B. 


$$
\bar{q}-q\left(x_{A}\right)-[u \phi]_{A}-\frac{h}{2} Q=0
$$

where $\bar{q}$ is the prescribed total flux at $x=L$ and $x_{A}=x_{B}-\frac{h}{2}$.

Using a second order expansion for the advective and diffusive fluxes at point $A$ gives [26]

$$
-u \phi+k \frac{\mathrm{d} \phi}{\mathrm{d} x}+\bar{q}-\frac{h}{2} r \quad \text { on } x=L
$$

where $r$ is given by Eq. (6). Again for $h=0$ the infinitesimal form of the 1D Neumann boundary condition is recovered.

It is important to recall that the underlined terms in Eqs. (5) and (8) introduce the necessary stabilization in the discrete solution using whatever numerical scheme [26-36].

Quite generally the FIC equation can be written for any problem in mechanics as $[26,27,46]$

$$
r_{i}-\frac{h_{i j}}{2} \frac{\partial r_{i}}{\partial x_{j}}=0, \quad \begin{aligned}
& i=1, n_{b} \\
& =1, n_{d}
\end{aligned}
$$

where $r_{i}$ is the $i$ th standard differential equation of the infinitesimal theory, $h_{i j}$ are characteristic length parameters; $n_{b}$ and $n_{d}$ are, respectively, the number of balance equations and the number of space dimensions of the problem (i.e., $n_{d}=2$ for $2 \mathrm{D}$ problems). The usual sum convention for repeated indexes is used in the text unless otherwise specified.

\section{Interpretation of the discrete solution of the FIC equations}

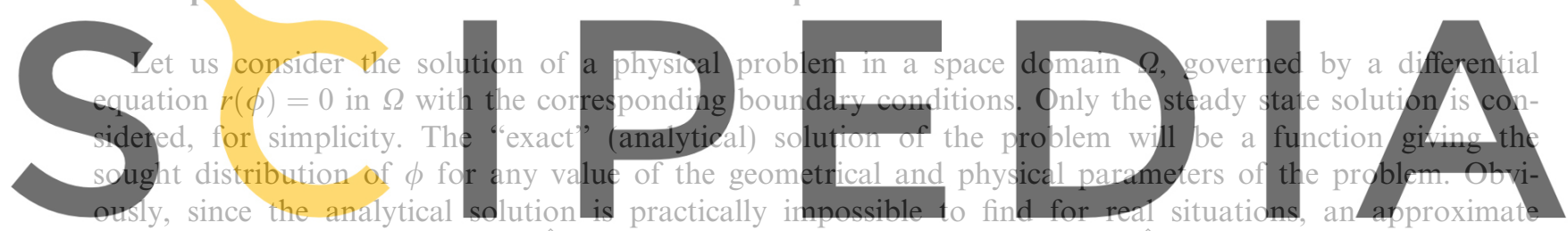

numerical solution is found $\phi \simeq \hat{\phi}$ by solving the problem $\hat{r}=0$, with $\hat{r}=r(\hat{\phi})$, using a particular discret-

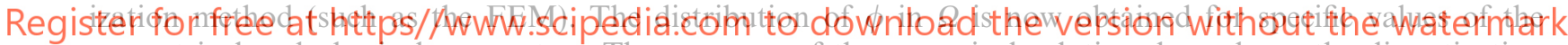
geometrical and physical parameters. The accuracy of the numerical solution depends on the discretization features, such as the number of elements and the approximating functions chosen in the FEM. Fig. 3 shows a schematic representation of the distribution of $\hat{\phi}$ along a line for different discretizations $M_{1}, M_{2}, \ldots, M_{n}$ where $M_{1}$ and $M_{n}$ are the coarser and finer meshes, respectively. Obviously for $n$ being sufficiently large a good approximation of $\phi$ will be obtained and for $M_{\infty}$ the approximate numerical solution $\hat{\phi}$ will coincide with the "exact" (and probably unreachable) analytical solution $\phi$ at all points. Indeed in some problems the $M_{\infty}$ solution can be found by a "clever" choice of the discretization parameters.

An unstable solution will occur when for some (typically coarse) discretizations, the numerical solution provides non-physical or very unaccurate values of $\hat{\phi}$. A situation of this kind is represented by curves $M_{1}$ and $M_{2}$ of the left hand side of Fig. 3. These unstabilities will disappear by an appropriate mesh refinement (curves $M_{3}, M_{4}, \ldots$ in Fig. 3) at the obvious increase of the computational cost.

In the FIC formulation the starting point are the modified differential equations of the problem as previously described. These equations are however not useful to find an analytical solution, $\phi(x)$, for the physical problem. Nevertheless, the numerical solution of the FIC equation can be readily found. Moreover, by adequately choosing the values of the characteristic length parameter $h$, the numerical solution of the FIC equations will be always stable (physically sound) for any discretization level chosen.

This process is schematically represented in Fig. 3 where it is shown that the numerical oscillations for the coarser discretizations $M_{1}$ and $M_{2}$ disappear when using the FIC procedure.

We can conclude the FIC approach allows us to obtain a better numerical solution for a given discretization. Indeed, as in the standard infinitesimal case, the choice of $M_{\infty}$ will yield the "exact" analytical solution and this ensures the consistency of the method. 


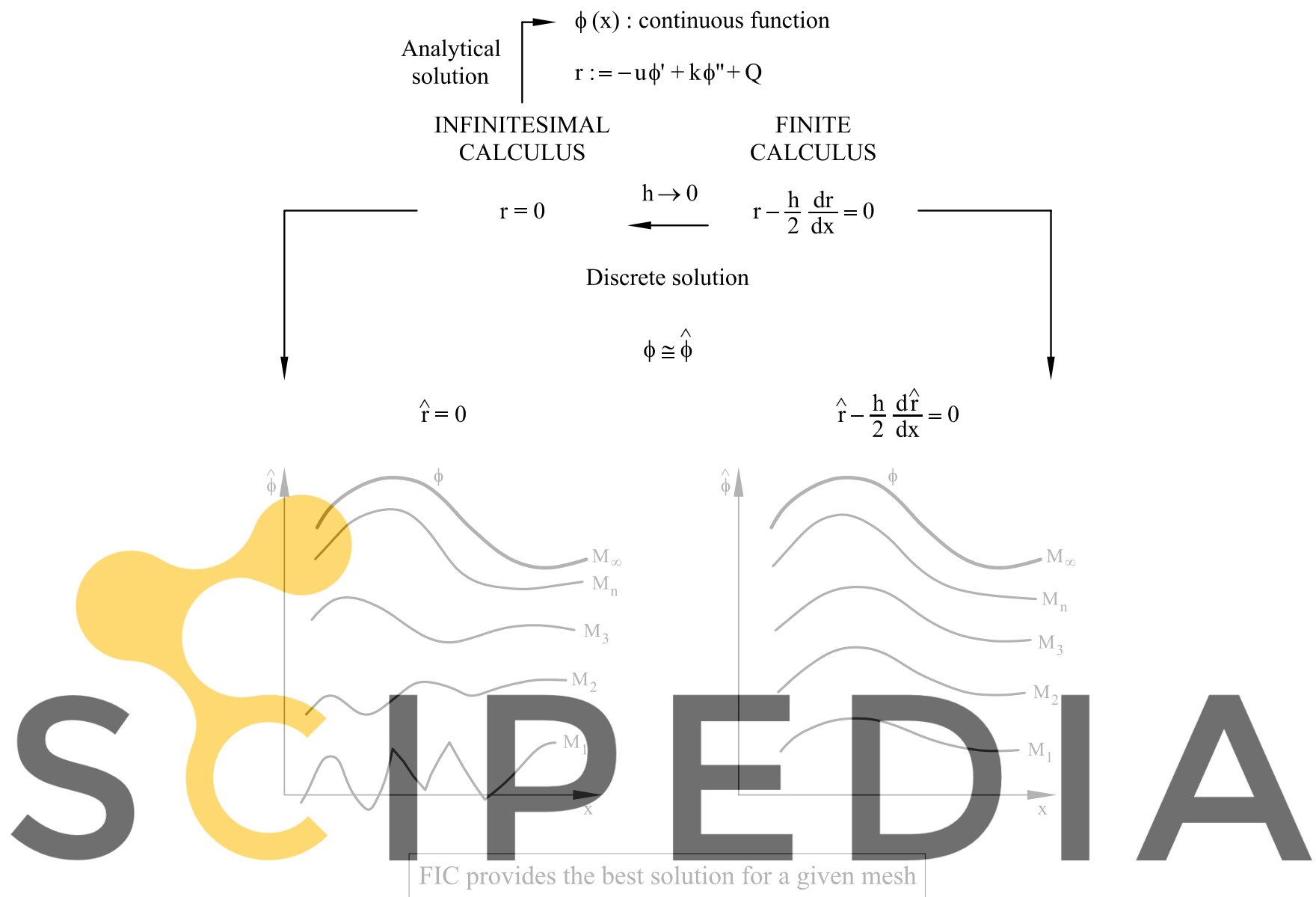

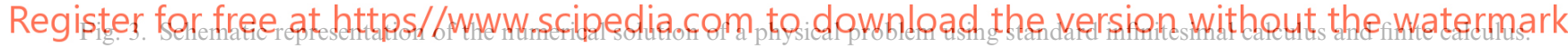

\section{FIC equations for incompressible flow}

The FIC governing equations for a viscous incompressible fluid can be written in an Eulerian frame of reference as (for simplicity we neglect the time stabilization terms) [46]

Momentum:

$$
r_{m_{i}}-\frac{1}{2} h_{i j} \frac{\partial r_{m_{i}}}{\partial x_{j}}=0 \text { in } \Omega \text { no sum in } i
$$

Mass balance:

$$
r_{d}-\frac{1}{2} h_{j} \frac{\partial r_{d}}{\partial x_{j}}=0 \quad \text { in } \Omega
$$

where

$$
\begin{aligned}
& r_{m_{i}}=\rho\left(\frac{\partial u_{i}}{\partial t}+u_{j} \frac{\partial u_{i}}{\partial x_{j}}\right)+\frac{\partial p}{\partial x_{i}}-\frac{\partial s_{i j}}{\partial x_{j}}-b_{i} \\
& r_{d}=\frac{\partial u_{i}}{\partial x_{i}} \quad i, j=1, n_{d}
\end{aligned}
$$


Above $\Omega$ is the analysis domain, $u_{i}$ is the velocity along the ith global axis, $\rho$ is the (constant) density of the fluid, $p$ is the absolute pressure (defined positive in compression), $b_{i}$ are the body forces and $s_{i j}$ are the viscous deviatoric stresses related to the viscosity $\mu$ by the standard expression

$$
s_{i j}=2 \mu\left(\dot{\varepsilon}_{i j}-\delta_{i j} \frac{1}{3} \frac{\partial u_{k}}{\partial x_{k}}\right)
$$

where $\delta_{i j}$ is the Kronecker delta and the strain rates $\dot{\varepsilon}_{i j}$ are

$$
\dot{\varepsilon}_{i j}=\frac{1}{2}\left(\frac{\partial u_{i}}{\partial x_{j}}+\frac{\partial u_{j}}{\partial x_{i}}\right)
$$

The FIC boundary conditions are

$$
\begin{aligned}
& n_{j} \sigma_{i j}-t_{i}+\frac{\frac{1}{2} h_{i j} n_{j} r_{m_{i}}}{\underline{n}}=0 \text { on } \Gamma_{t} \text { no sum in } i \\
& u_{j}-u_{j}^{p}=0 \quad \text { on } \Gamma_{u}
\end{aligned}
$$

and the initial condition is $u_{j}=u_{j}^{0}$ for $t=t_{0}$.

In Eqs. (16) and (17) $t_{i}$ and $u_{j}^{p}$ are surface tractions and prescribed velocities on the boundaries $\Gamma_{t}$ and $\Gamma_{u}$, respectively, $n_{j}$ are the components of the unit normal vector to the boundary and $\sigma_{i j}$ are the total stresses given by $\sigma_{i j}=s_{i j}-\delta_{i j} p$.

Distances $h_{i}$ in Eq. (11) denote the dimensions of the domain where balance of mass is enforced. This is a basic difference with the momentum equations where the momentum balance law is applied along each global coordinate direction, thereby introducing the characteristic lengths $h_{i j}$ in Eq. (10) [46]. In Eq. (16) the $h_{i j}$ 's define the domain where equilibrium of boundary tractions is established [26,46]. As mentioned earlier, in

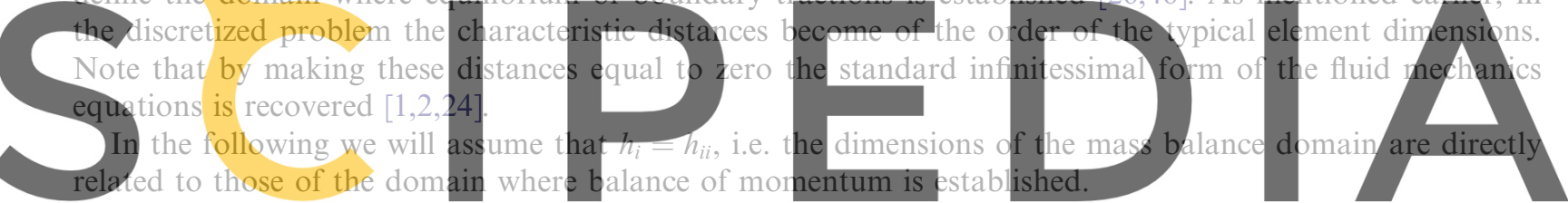

Eqs. (10)-(17) are the starting point for deriving stabilized FEM for solving the incompressible Navier-

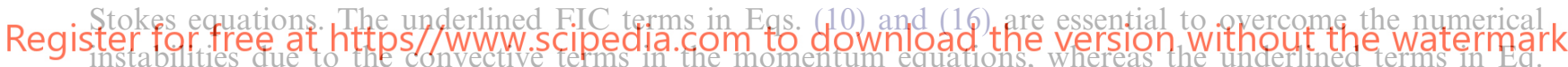
(11) take care of the instabilities due to the incompressibility constraint. An interesting feature of the FIC formulation is that it aliows to use equal order interpolation for the velocity and pressure variables [37-49].

\subsection{Stabilized integral forms}

From the momentum equations it can be obtained (for $\left.h_{i}=h_{i i}\right)[37,45,46]$

$$
\frac{\partial r_{d}}{\partial x_{i}} \simeq \frac{h_{i} i}{2 a_{i}} \frac{\partial r_{m_{i}}}{\partial x_{j}}, \quad \text { no sum in } i
$$

where

$$
a_{i}=\frac{2 \mu}{3}+\frac{\rho u_{i} h_{i i}}{2}, \text { no sum in } i
$$

Substituting Eq. (18a) into Eq. (11) and retaining the terms involving the derivatives of $r_{m_{i}}$ with respect to $x_{i}$ only, leads to the following alternative expression for the stabilized mass balance equation

$$
r_{d}-\sum_{i=1}^{n_{d}} \tau_{i} \frac{\partial r_{m_{i}}}{\partial x_{i}}=0
$$

with

$$
\left.\tau_{i}=\frac{8 \mu}{3 h_{i i}^{2}}+\frac{2 \rho u_{i}}{h_{i i}}\right)^{-1}
$$


The $\tau_{i}$ 's in Eq. (19) when multiplied by the density are equivalent to the intrinsic time parameters, seen extensively in the stabilization literature. The interest of Eq. (19) is that it introduces the first space derivatives of the momentum equations into the mass balance equation. These terms have intrinsic good stability properties as explained next.

The weighted residual forms of the momentum and mass balance equations (Eqs. (10) and (19)) are written as

$$
\begin{aligned}
& \int_{\Omega} \delta u_{i}\left[r_{m_{i}}-\frac{h_{i j}}{2} \frac{\partial r_{m_{i}}}{\partial x_{j}}\right] \mathrm{d} \Omega+\int_{\Gamma_{t}} \delta u_{i}\left(\sigma_{i j} n_{j}-t_{i}+\frac{h_{i j}}{2} n_{j} r_{m_{i}}\right) \mathrm{d} \Gamma=0 \\
& \int_{\Omega} q\left[r_{d}-\sum_{i=1}^{n_{d}} \tau_{i} \frac{\partial r_{m_{i}}}{\partial x_{i}}\right] \mathrm{d} \Omega=0
\end{aligned}
$$

where $\delta u_{i}$ and $q$ are arbitrary weighting functions representing virtual velocities and virtual pressure fields. Integrating by parts the $r_{m_{i}}$ derivative terms in Eqs. (21) and (22) yields

$$
\begin{aligned}
& \int_{\Omega} \delta u_{i} r_{m_{i}} \mathrm{~d} \Omega+\int_{\Gamma_{t}} \delta u_{i}\left(\sigma_{i j} n_{j}-t_{i}\right) \mathrm{d} \Gamma+\int_{\Omega} \frac{h_{i j}}{2} \frac{\partial \delta u_{i}}{\partial x_{j}} r_{m_{i}} \mathrm{~d} \Omega=0 \\
& \int_{\Omega} q r_{d} \mathrm{~d} \Omega+\int_{\Omega}\left[\sum_{i=1}^{n_{d}} \tau_{i} \frac{\partial q}{\partial x_{i}} r_{m_{i}}\right] \mathrm{d} \Omega-\int_{\Gamma}\left[\sum_{i=1}^{n_{d}} q \tau_{i} n_{i} r_{m_{i}}\right] \mathrm{d} \Gamma=0
\end{aligned}
$$

We will neglect hereonwards the third integral in Eq. (23b) by assuming that $r_{m_{i}}$ is negligible on the bound-

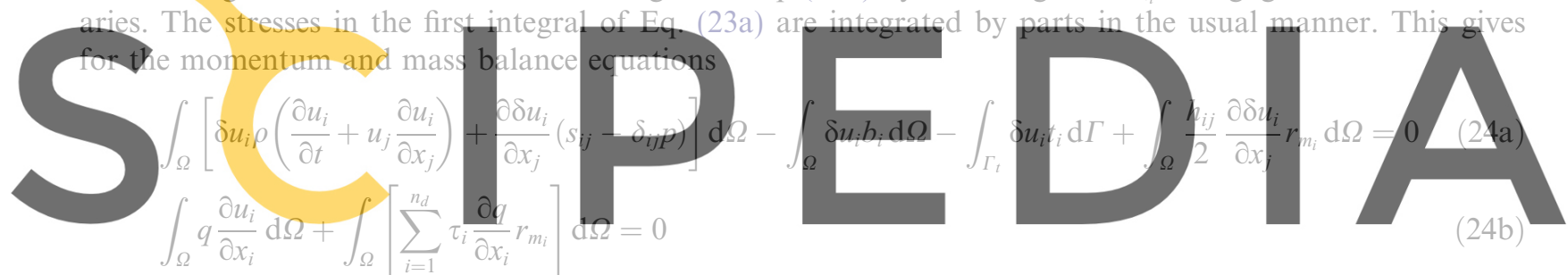

Register for free at https//www.scipedia.com to download the version without the watermark

\subsection{Convective and pressure gradient projections}

The computation of the residual terms are simplified if we introduce the convective and pressure gradient projections $c_{i}$ and $\pi_{i}$, respectively, defined as

$$
\begin{aligned}
& c_{i}=r_{m_{i}}-\rho u_{j} \frac{\partial u_{i}}{\partial x_{j}} \\
& \pi_{i}=r_{m_{i}}-\frac{\partial p}{\partial x_{i}}
\end{aligned}
$$

We can express $r_{m_{i}}$ in Eqs. (24) in terms of $c_{i}$ and $\pi_{i}$, respectively which then become additional variables. The system of integral equations is now augmented in the necessary number of equations by imposing that the residual $r_{m_{i}}$ vanishes (in average sense) for both forms given by Eqs. (25). This gives the final system of governing equation as:

$$
\begin{gathered}
\int_{\Omega}\left[\delta u_{i} \rho\left(\frac{\partial u_{i}}{\partial t}+u_{j} \frac{\partial u_{i}}{\partial x_{j}}\right)+\frac{\partial \delta u_{i}}{\partial x_{j}}\left(s_{i j}-\delta_{i j} p\right)\right] \mathrm{d} \Omega-\int_{\Omega} \delta u_{i} b_{i} \mathrm{~d} \Omega \\
\quad-\int_{\Gamma_{t}} \delta u_{i} t_{i} \mathrm{~d} \Gamma+\int_{\Omega} \frac{h_{i k}}{2} \frac{\partial\left(\delta u_{i}\right)}{\partial x_{k}}\left(\rho u_{j} \frac{\partial u_{i}}{\partial x_{j}}+c_{i}\right) \mathrm{d} \Omega=0 \\
\int_{\Omega} q \frac{\partial u_{i}}{\partial x_{i}} \mathrm{~d} \Omega+\int_{\Omega} \sum_{i=1}^{n_{d}} \tau_{i} \frac{\partial q}{\partial x_{i}}\left(\frac{\partial p}{\partial x_{i}}+\pi_{i}\right) \mathrm{d} \Omega=0
\end{gathered}
$$




$$
\begin{aligned}
& \int_{\Omega} \delta c_{i} \rho\left(\rho u_{j} \frac{\partial u_{i}}{\partial x_{j}}+c_{i} \mathrm{~d} \Omega=0 \quad \text { no sum in } i\right. \\
& \int_{\Omega} \delta \pi_{i} \tau_{i}\left(\frac{\partial p}{\partial x_{i}}+\pi_{i}\right) \mathrm{d} \Omega=0 \quad \text { no sum in } i
\end{aligned}
$$

with $i, j, k=1, n_{d}$. In Eqs. (28) and (29) $\delta c_{i}$ and $\delta \pi_{i}$ are appropriate weighting functions and the $\rho$ and $\tau_{i}$ weights are introduced for convenience.

The convective and pressure gradient projections enforce the consistency of the formulation as it ensures that the stabilization terms in Eqs. (26) and (27) have a residual form which vanishes for the "exact" solution. Neglecting these terms reduces the accuracy of the numerical solution and it makes the formulation more sensitive to the value of the stabilization parameters $[45,47,48]$.

\section{Finite element solution of FIC equations for incompressible flow}

We choose $C^{\circ}$ continuous linear interpolations of the velocities, the pressure, the convection projections $c_{i}$ and the pressure gradient projections $\pi_{i}$ over 3-noded triangles (2D) and 4-noded tetrahedra (3D). The linear interpolations are written as

$$
\begin{array}{ll}
u_{i}=N^{k} \bar{u}_{i}^{k}, & p=N^{k} \bar{p}^{k} \\
c_{i}=N^{k} \bar{c}_{i}^{k}, & \pi_{i}=N^{k} \bar{\pi}_{i}^{k}
\end{array}
$$

where the sum goes over the number of nodes of each element $n\left(n=3 / 4\right.$ for triangles/tetrahedra), $(\cdot)^{k}$ denotes the nodal variables and $N^{k}$ are the linear shape functions $[1,24]$.
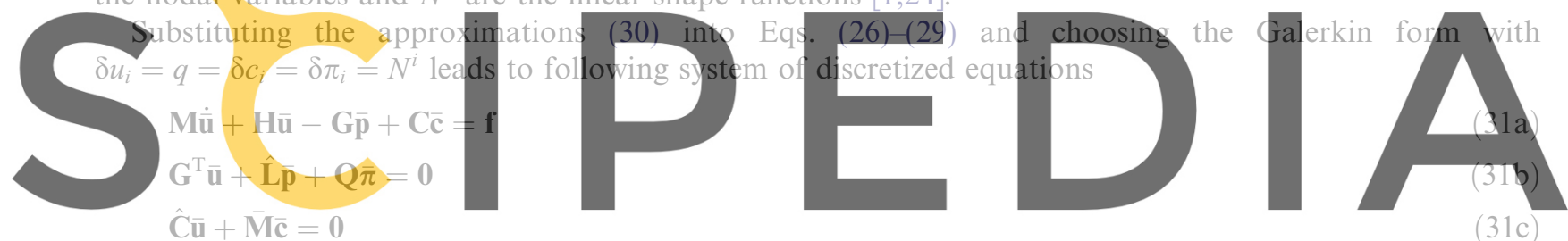

(31c)

Register fQr freêlaththtps//www.scipedia.com to download the version without the waterphilk

where

$$
\mathbf{H}=\mathbf{A}+\mathbf{K}+\hat{\mathbf{K}}
$$

If we denote the node indexes with superscripts $a, b$ and the space indices with subscripts $i, j$, the element contributions to the components of the arrays involved in these equations are (some expressions are explicitly given for 2D problems)

$$
\begin{aligned}
& M_{i j}^{a b}=\left(\int_{\Omega^{\mathrm{e}}} \rho N^{a} N^{b} \mathrm{~d} \Omega\right) \delta_{i j}, \quad A_{i j}^{a b}=\left(\int_{\Omega^{\mathrm{e}}} \rho N^{a}\left(\mathbf{u}^{\mathrm{T}} \nabla N^{b}\right) \mathrm{d} \Omega\right) \delta_{i j} \\
& \mathbf{K}^{a b}=\int_{\Omega^{\mathrm{e}}} \mathbf{B}_{a}^{\mathrm{T}} \mathbf{D} \mathbf{B}_{b} \mathrm{~d} \Omega, \quad \mathbf{B}_{a}=\left[\begin{array}{ll}
\frac{\partial N^{a}}{\partial x_{1}} & 0 \\
0 & \frac{\partial N^{a}}{\partial x_{2}} \\
\frac{\partial N^{a}}{\partial x_{2}} & \frac{\partial N^{a}}{\partial x_{1}}
\end{array}\right], \quad \mathbf{D}=\mu\left[\begin{array}{lll}
4 / 3 & -2 / 3 & 0 \\
-2 / 3 & 4 / 3 & 0 \\
0 & 0 & 1
\end{array}\right] \\
& \hat{K}_{i j}^{a b}=\left(\frac{1}{2} \int_{\Omega^{\mathrm{e}}} h_{i j} \frac{\partial N^{a}}{\partial x_{j}}\left(\rho \mathbf{u}^{\mathrm{T}} \nabla N^{b}\right) \mathrm{d} \Omega\right) \delta_{i j}, \quad \mathbf{G}=\left\{\begin{array}{l}
\mathbf{G}_{1} \\
\mathbf{G}_{2}
\end{array}\right\}, \quad G_{i}^{a b}=\int_{\Omega^{\mathrm{e}}} \frac{\partial N^{a}}{\partial x_{i}} N^{b} \mathrm{~d} \Omega \\
& \mathbf{C}=\left[\begin{array}{l}
\mathbf{C}^{1} \\
\mathbf{C}^{2}
\end{array}\right], \quad C_{i}^{a b}=\frac{1}{2} \int_{\Omega^{\mathrm{e}}} h_{i j} \frac{\partial N^{a}}{\partial x_{j}} N^{b} \mathrm{~d} \Omega, \quad \bar{M}^{a b}=\int_{\Omega^{\mathrm{e}}} \rho N^{a} N^{b} \mathrm{~d} \Omega \\
& \hat{L}^{a b}=\int_{\Omega^{\mathrm{e}}}\left(\nabla^{\mathrm{T}} N^{a}\right)[\tau] \nabla N^{b} \mathrm{~d} \Omega, \quad[\tau]=\left[\begin{array}{ll}
\tau_{1} & 0 \\
0 & \tau_{2}
\end{array}\right]
\end{aligned}
$$




$$
\begin{aligned}
& \mathbf{Q}=\left[\mathbf{Q}_{1}, \mathbf{Q}_{2}\right], \quad Q_{i}^{a b}=\int_{\Omega^{\mathrm{e}}} \tau_{i} \frac{\partial N^{a}}{\partial x_{i}} N^{b} \mathrm{~d} \Omega \quad \text { no sum in } i \\
& \hat{\mathbf{C}}^{\mathrm{T}}=\left[\hat{\mathbf{C}}_{1}, \hat{\mathbf{C}}_{2}\right], \quad \hat{C}_{1}^{a b}=\hat{C}_{2}^{a b}=\int_{\Omega^{\mathrm{e}}} \rho^{2} N^{a}\left(\mathbf{u}^{\mathrm{T}} \nabla N^{b}\right) \mathrm{d} \Omega \\
& \hat{\mathbf{M}}^{\mathrm{T}}=\left[\hat{\mathbf{M}}^{1}, \hat{\mathbf{M}}^{2}\right], \quad \hat{M}_{i j}^{a b}=\left(\int_{\Omega^{\mathrm{e}}} \tau_{i} N^{a} N^{b} \mathrm{~d} \Omega\right) \delta_{i j}, \quad f_{i}^{a}=\int_{\Omega^{\mathrm{e}}} N^{a} f_{i} \mathrm{~d} \Omega+\int_{\Gamma^{\mathrm{e}}} N^{a} t_{i} \mathrm{~d} \Gamma
\end{aligned}
$$

It is understood that all the arrays are matrices (except $\mathbf{f}$ which is a vector) whose components are obtained by grouping together the left indices in the previous expressions ( $a$ and possibly $i$ ) and the right indices $(b$ and possibly $j$ ).

Note that the stabilization matrix $\hat{\mathbf{K}}$ in Eq. (33) adds new orthotropic diffusivity terms of value $\rho \frac{h_{i j} u_{l}}{2}$. A discussions of these terms is presented in [54].

The overall stabilization terms introduced by the FIC formulation have the intrinsic capacity to ensure physically sound numerical solutions for a wide spectrum of Reynolds numbers without the need of introducing additional turbulence modelling terms. This property is validated in the examples presented in a next section.

\subsection{Transient solution scheme}

The solution in time of the system of Eqs. (31) can be written in general form as

$$
\mathbf{M} \frac{1}{\Delta t}\left(\overline{\mathbf{u}}^{n+1}-\overline{\mathbf{u}}^{n}\right)+\mathbf{H}^{n+\theta} \overline{\mathbf{u}}^{n+\theta}-\mathbf{G} \overline{\mathbf{p}}^{n+\theta}+\mathbf{C}^{n+\theta} \overline{\mathbf{c}}^{n+\theta}=\mathbf{f}^{n+\theta}
$$

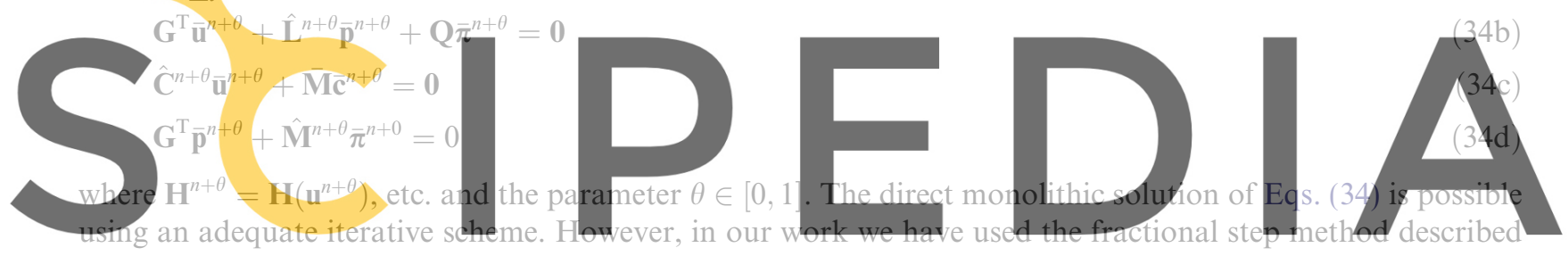

in $[45,46]$.

Register for free at https//www.scipedia.com to download the version without the watermark

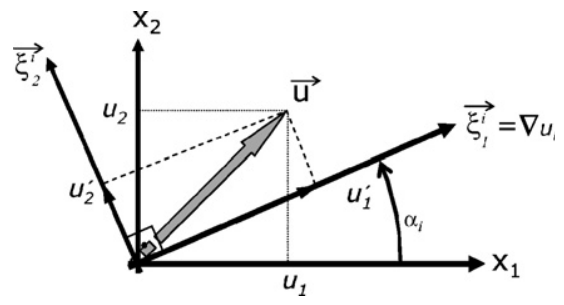

Fig. 4. Definition of the principal curvature direction $\vec{\xi}_{1}^{i}$ along the gradient of $u_{i}$.

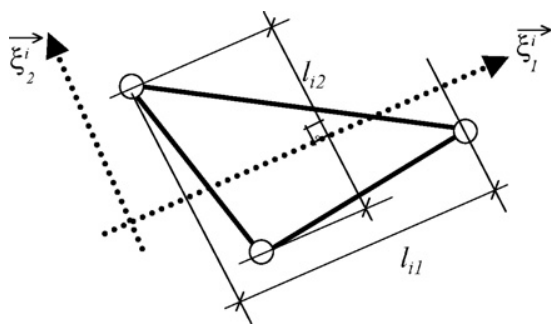

Fig. 5. Definition of the element characteristic distances $l_{i 1}$ and $l_{i 2}$ corresponding to the $i$ th momentum equation. 


\section{Computation of the characteristic distances}

The computation of the stabilization parameters is a crucial issue as they affect both the stability and accuracy of the numerical solution. The different procedures to compute the stabilization parameters are typically based on the study of simplified forms of the stabilized equations. Contributions to this topic are reported in [12-22,27-36,45-51].

Recent work of the authors has shown that the stabilizing FIC terms for convection-diffusion problems take the form of a simple orthotropic diffusion if the balance equation is written in the principal curvature directions of the solution. Excellent results were reported in $[33,35]$ by computing first the characteristic length distances along the principal curvature directions, followed by a standard transformation of the distances to global axes. The resulting stabilized finite element equations capture the high gradient zones in the vicinity of the domain edges (boundary layers) as well as the sharp gradients appearing randomly in the interior of the domain $[33,35]$. The FIC/FEM thus reproduces the best features of the so called transverse (cross-wind) dissipation or shock capturing methods $[1,2,24]$.

The numerical computations are simplified without apparent loss of accuracy if the main principal curvature direction of the solution at each element point is approximated by the direction of the gradient vector at the element center $[33,35]$. The other principal directions are taken in orthogonal directions to the gradient. For linear triangles and quadrilaterals these directions are assumed to be constant within the element.

Above simple scheme has been used in this work for the computation of the characteristic distances $h_{i j}$. Details of the algorithm are given next. The method is explained for $2 \mathrm{D}$ problems although it is readily extendible to $3 \mathrm{D}$ problems [54].

For the $i$ th momentum balance equation and every time step of the transient solution scheme:

1. A coordinate system $\vec{\xi}_{1}^{i}, \vec{\xi}_{2}^{i}$ is defined at each element point such that $\vec{\xi}_{1}^{i}$ is aligned with the gradient of $u_{i}$ $\left(\vec{\xi}_{1}^{i}=\nabla \vec{u}_{i}\right)$ and $\vec{\xi}_{2}^{i}$ is orthogonal to $\vec{\xi}_{1}^{i}$ in anticlockwise sense (Fig. 4). The angle that $\vec{\xi}_{1}^{i}$ forms with the global $x_{1}$ axis is defined as $\alpha_{i}$. Recall that upper and lower index $i$ denotes the $i$ th momentum equation.

2. The element characteristic distances $l_{i 1}$ and $l_{i 2}$ are defined as the maximum projections of the element sides along the $\vec{\xi}_{1}^{i}$ and $\vec{\xi}_{2}^{i}$ axes, respectively (Fig. 5).

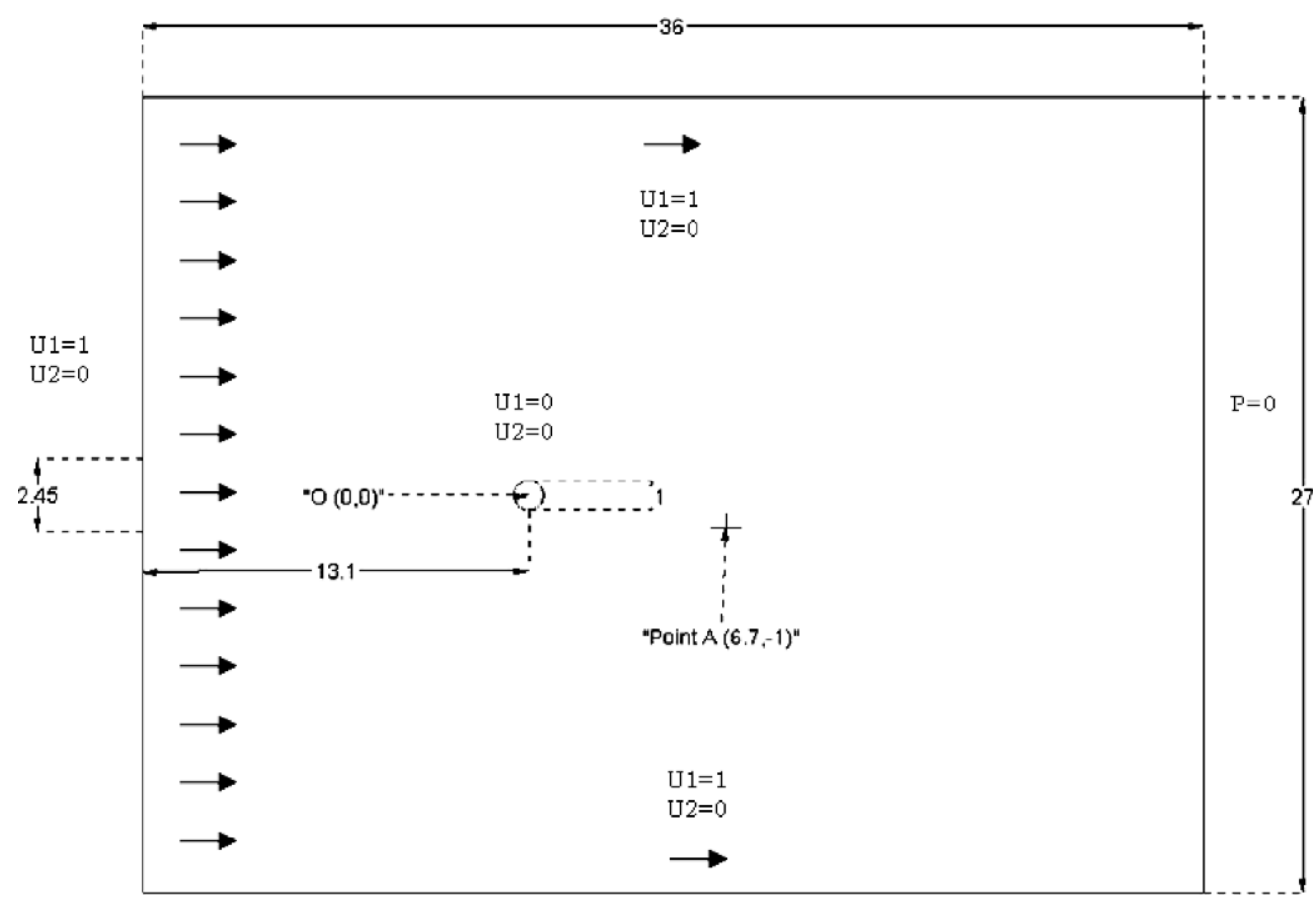

Fig. 6. Flow past a cylinder of unit diameter. Analysis domain and boundary conditions. 
3. The characteristic distances $h_{i 1}$ and $h_{i 2}$ are computed as

$$
\left\{\begin{array}{l}
h_{i 1} \\
h_{i 2}
\end{array}\right\}=\left[\begin{array}{ll}
c_{i} & -s_{i} \\
s_{i} & c_{i}
\end{array}\right]\left\{\begin{array}{l}
h_{i 1}^{\prime} \\
h_{i 2}^{\prime}
\end{array}\right\}, \quad i=1,2
$$

with $c_{i}=\cos \alpha_{i}, s_{i}=\sin \alpha_{i}$ and the local distances $h_{i 1}^{\prime}$ and $h_{i 2}^{\prime}$ are

$$
\left.h_{i j}^{\prime}=\operatorname{coth} \gamma_{i j}-\frac{1}{\gamma_{i j}}\right) l_{i j}, \quad \gamma_{i j}=\frac{u_{j}^{\prime} l_{i j}}{2 \mu}, \quad j=1,2
$$

where $u_{1}^{\prime}$ and $u_{2}^{\prime}$ are the components of the velocity vector along the local axes $\vec{\xi}_{1}^{i}$ and $\vec{\xi}_{2}^{i}$, respectively (Fig. 4).

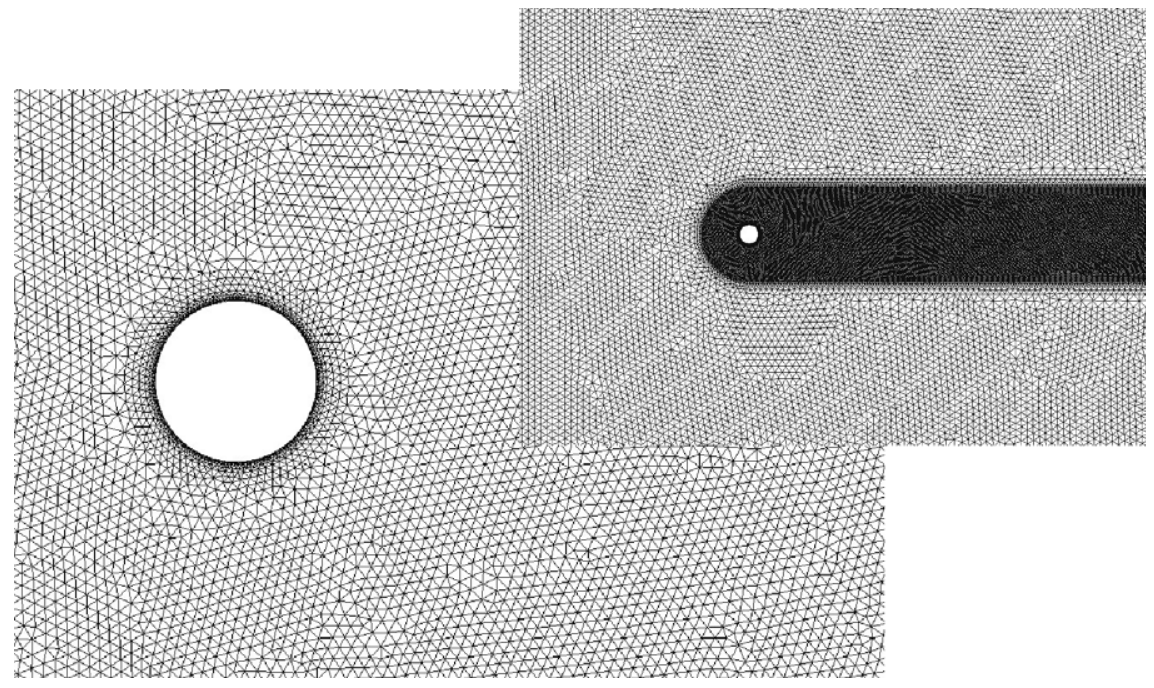

Fig. 7. Flow past a cylinder. Mesh of 59380 three-noded triangles used for the computations.

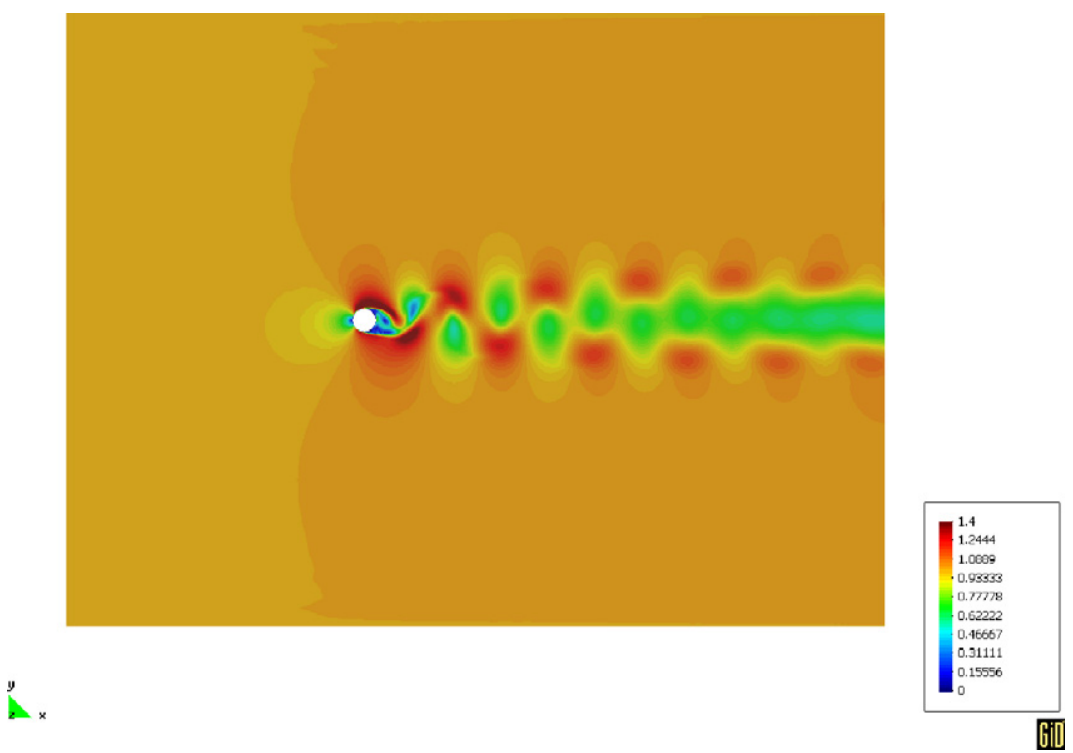

Fig. 8. Flow past a cylinder, $R e=1000$. Contour of the velocity vector module for $t=100 \mathrm{~s}$. 


\section{Examples}

The examples were solved with the Tdyn code where the formulation here presented has been implemented. The Tdyn code can be downloaded from the webpage given in [52].

\section{1. $2 D$ flow past a cylinder}

Fig. 6 shows the geometry for the analysis of the flow past a cylinder of unit diameter $(D)$. A unit horizontal velocity is prescribed at the inlet boundary and at the two horizontal walls. Zero pressure is prescribed at the outlet boundary. The dimensions of the analysis domain are $36 \times 27$ units. The origin of the coordinate system has been sampled at the center of the cylinder located at a distance of 13.1 units from the entry wall. Zero velocity is prescribed at the cylinder wall. The kinematic viscosity $\left(v=\frac{\mu}{\rho}\right)$ is $v=0.001$. Fig. 7 shows the mesh of 59,380 three-noded elements used for the computation (160 along its circumference) and 29,398 nodes. The radial thickness of the layer of elements around the cylinder is 0.02 . The number of elements in the highlyrefined inner mesh is 47,716 .

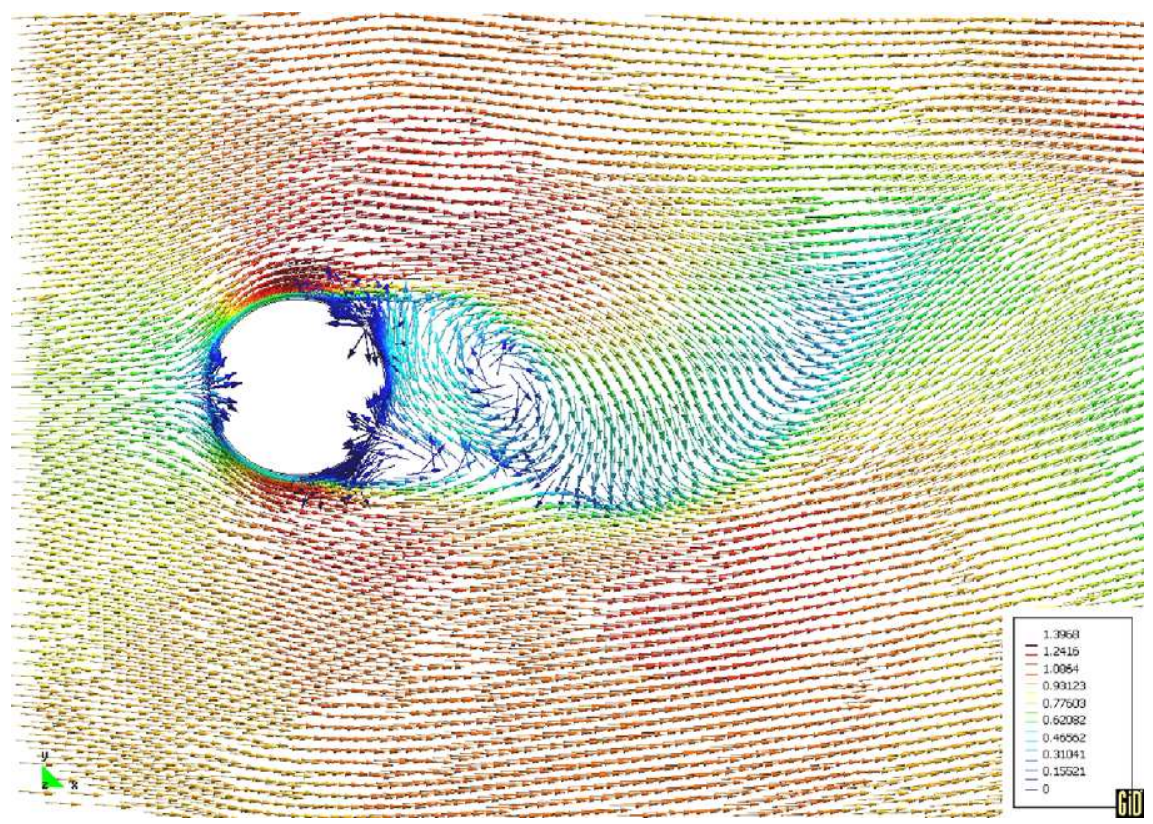

Fig. 9. Flow past a cylinder, $R e=1000$. Velocity vectors for $t=100 \mathrm{~s}$.

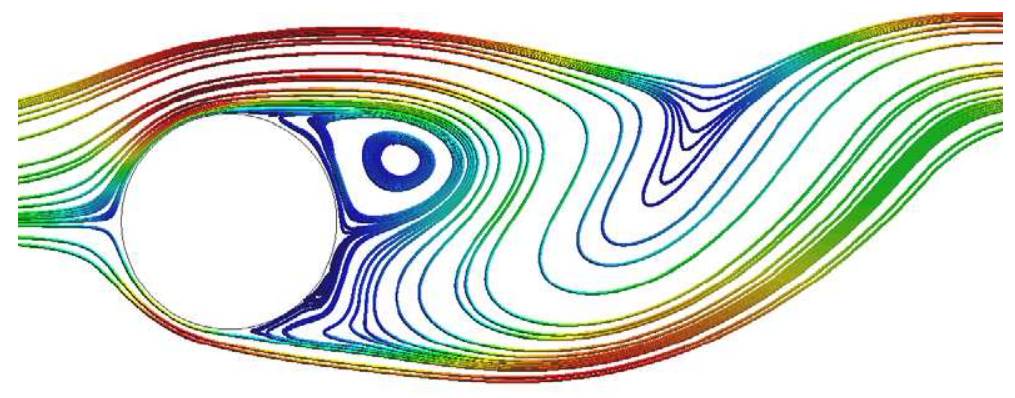

I.

Fig. 10. Streamlines around the cylinder at time $t=100 \mathrm{~s}$. 
The problem has been analyzed for a value of the horizontal velocity at the entry of $u_{1}=1$ giving a Reynolds number of $R e=1000$. The time step for the solution of Eqs. (34) using a fractional step method was set equal to $0.05 \mathrm{~s}[45,46]$. Figs. 8 and 9 respectively show the velocity modulus contours and the velocity vectors for $t=100 \mathrm{~s}$.

Fig. 10 shows the streamlines around the cylinder at time $t=100 \mathrm{~s}$. It is shown the two vortices created in the turbulent wake of the cylinder. Fig. 11 shows images of the trajectory of a substance over a band of 2.45 units transported at the entry across the flow for $t=100 \mathrm{~s}$. The picture shows clearly the oscillatory nature of the flow.
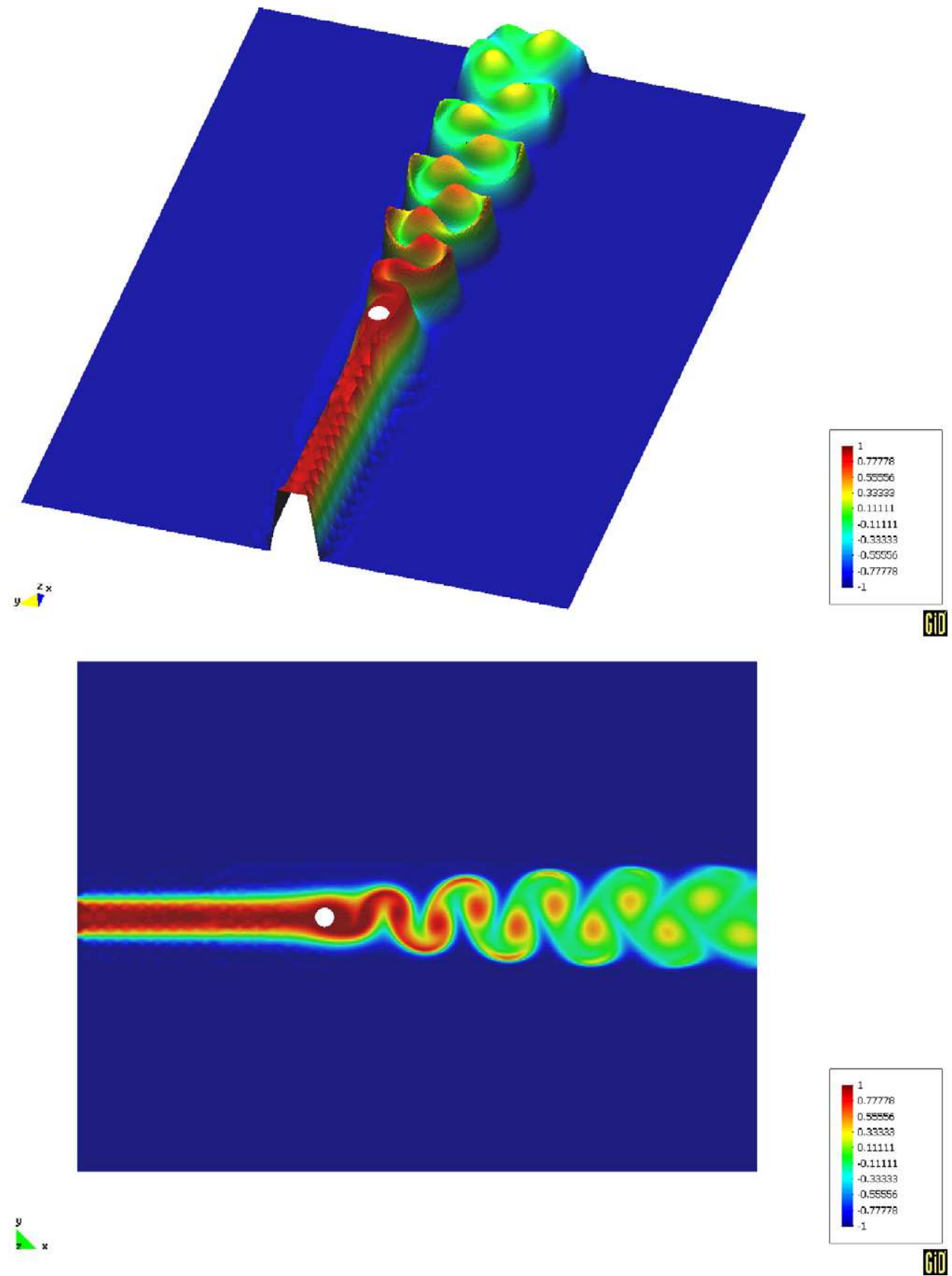

Fig. 11. Flow past a cylinder, $R e=1000$. Trajectories of a substance over a band of 2.45 units at the entry transported across the flow for $t=100 \mathrm{~s}$ 


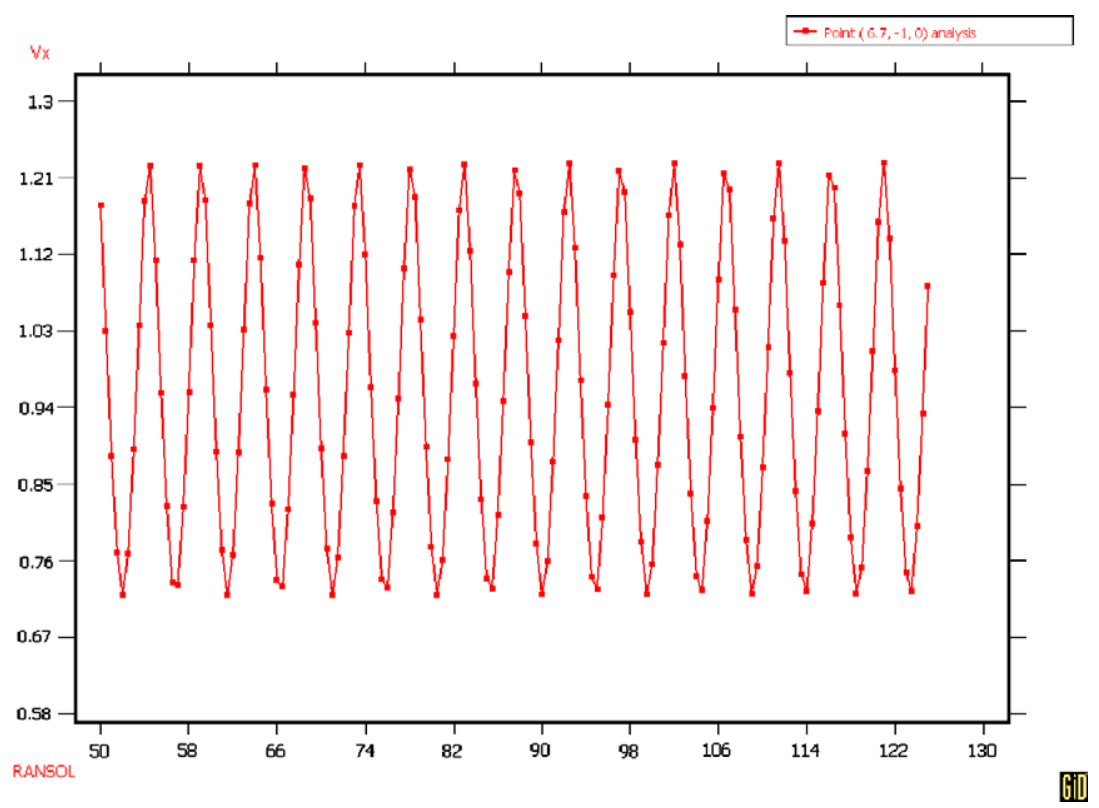

Fig. 12. 3D flow past a cylinder, $R e=1000$. Oscillations with time of the horizontal velocity at the point $A$ with coordinates (6.7-1.02).
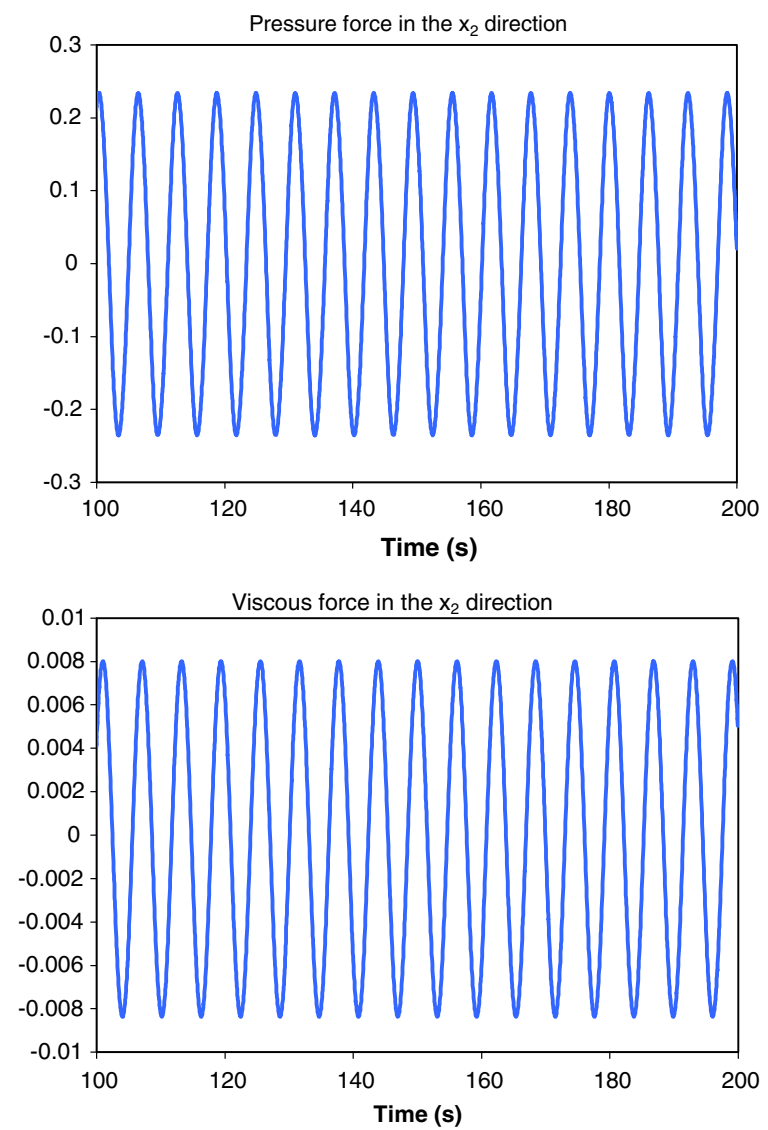

Fig. 13. Time histories of the pressure and the viscous forces along the vertical axis over the cylinder from time 50 to $200 \mathrm{~s}$. 
Fig. 12 shows the oscillations of the horizontal velocity at the point $A$ with coordinates $(6.7,-1.02)$ with time. Fig. 13 shows the time histories of the pressure and the viscous forces along the vertical axis over the cylinder from time 50 to $200 \mathrm{~s}$. The Strouhal number computed from the shedding frequency $n$ as $S=\frac{n D}{|\mathbf{u}|}$ is $S=0.2103$. This number compares very well with the experimental result available in the literature as shown in Fig. 14 where the Strouhal numbers obtained for $R e=1000$ and $R e=100$ are plotted. More details of these results can be found in [46].

It is a well known fact that for $R e>300$ the flow past a cylinder exhibits 3D features. In [53] results from 2D and 3D computations were compared for $R e=300$ and 800 . While 3D features were observed even at $R e=300$ and more so at $R e=800$, there were no large discrepances between the global flow parameters (such as drag, lift and Strouhal number) obtained from 2D and 3D computations. These conclusions justify the results of the $2 \mathrm{D}$ computations presented here.

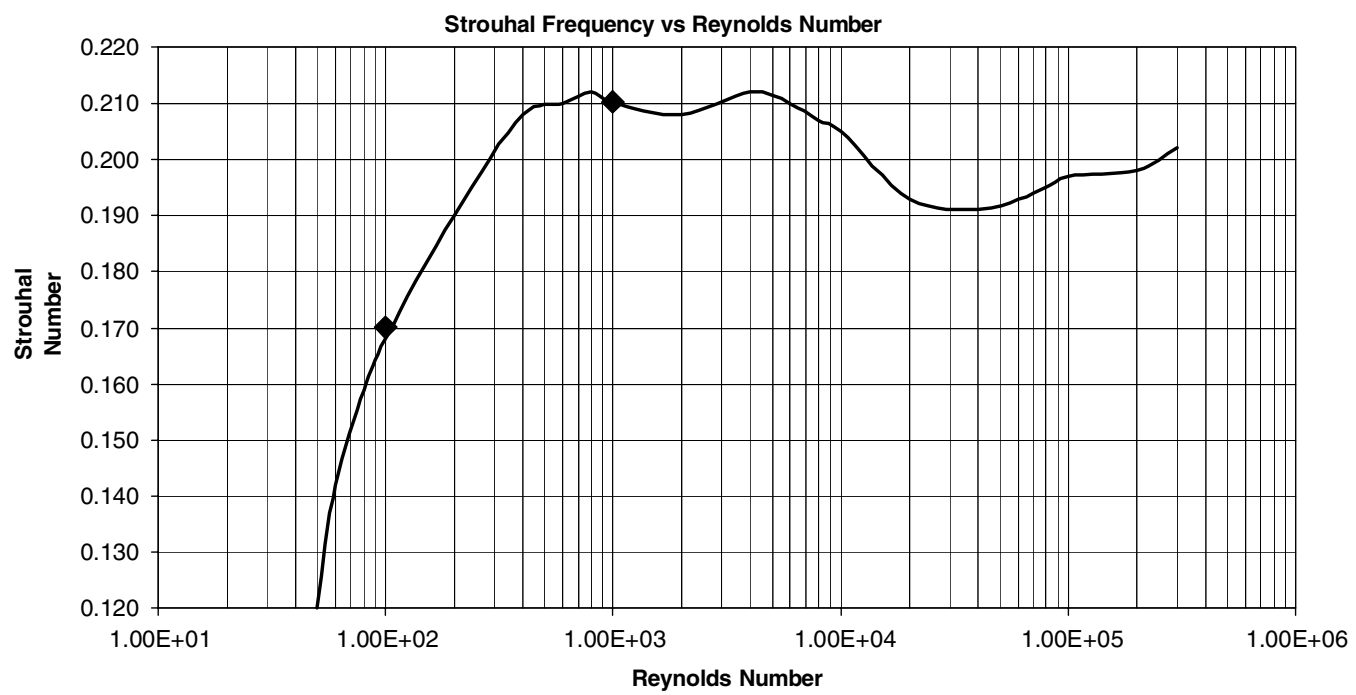

Fig. 14. Flow past a cylinder. Experimental (thick line) and computed (filled diamond) values of the Strouhal number $S$ in terms of the Reynolds number. Experimental values taken from [http://wn7.enseeiht.fr/hmf/travaux/CD0102/travaux/optmfn/gpfmho/01-2/grp1/ index.htm].
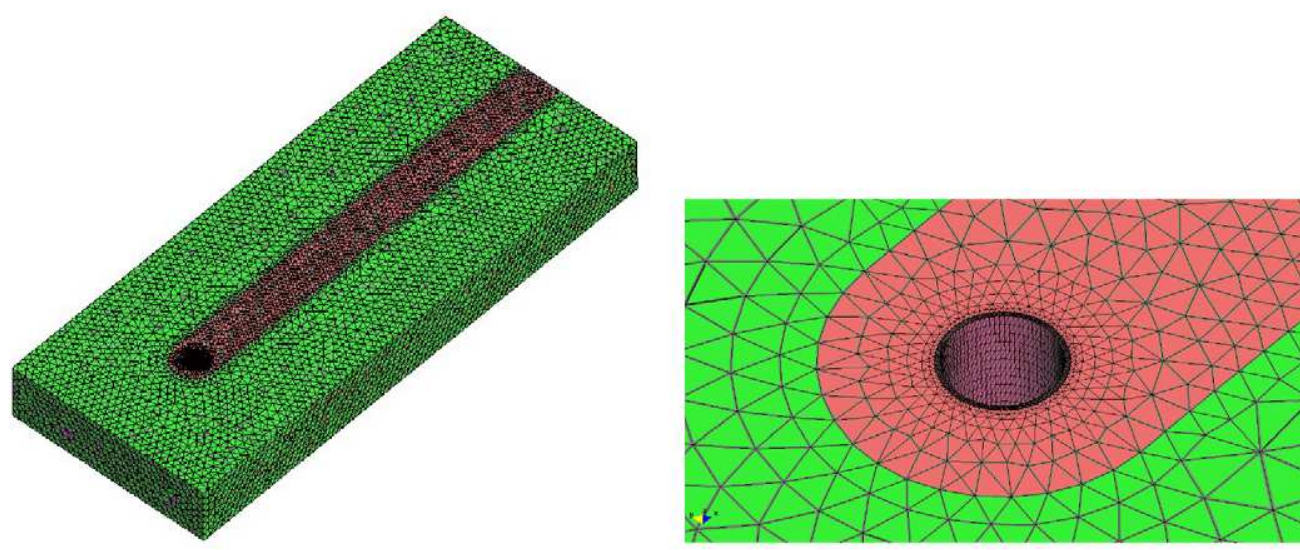

Fig. 15. Flow past a cylinder, $R e=400$. Mesh of 445814 four-noded tetrahedra used for the computations. 


\section{2. $3 D$ flow past a cylinder}

The 3D analysis of the flow about a cylinder was solved for $R e=400$. Geometry and boundary conditions are the natural extension of the 2D case presented in the previous section. The dimension of the computational domain parallel to the cylinder axis is 8 units (this length is recommended in [53] to capture a few wavelengths along the cylinder axis). The boundary conditions for the lateral boundary perpendicular to the cylinder axis consist of zero-normal velocity and zero-shear stress. Fig. 15 shows the mesh of 445,814 four-noded tetrahedra used for the computation (40 elements along the cylinder span and 80 elements along its circumference) and

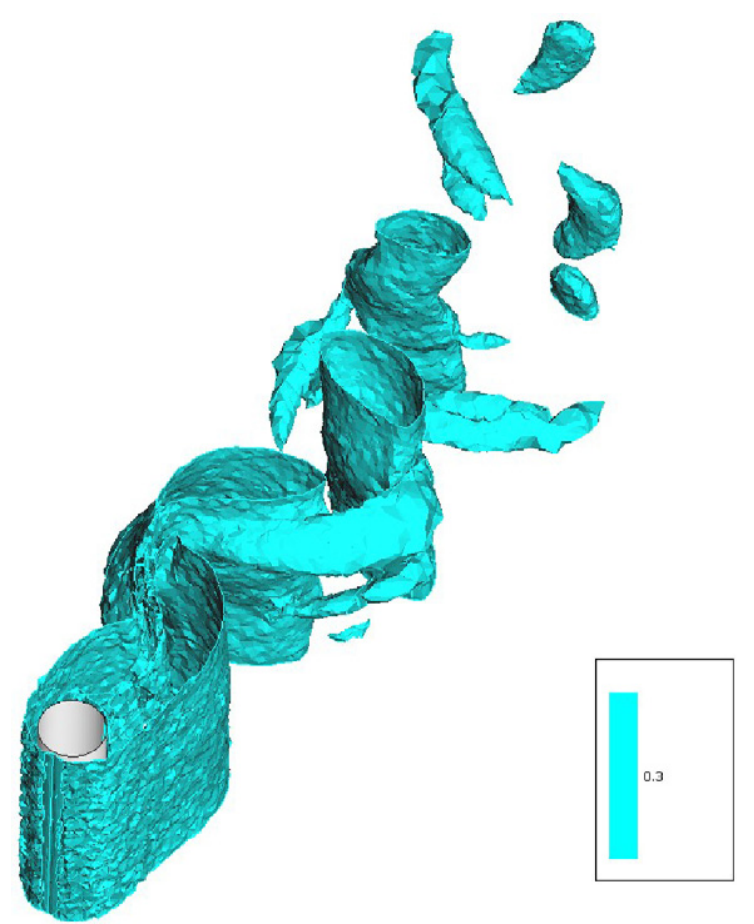

Fig. 16. $3 \mathrm{D}$ cylinder. Iso-contours of the vorticity modulus $(w=0.3)$ at $t=100 \mathrm{~s}$.

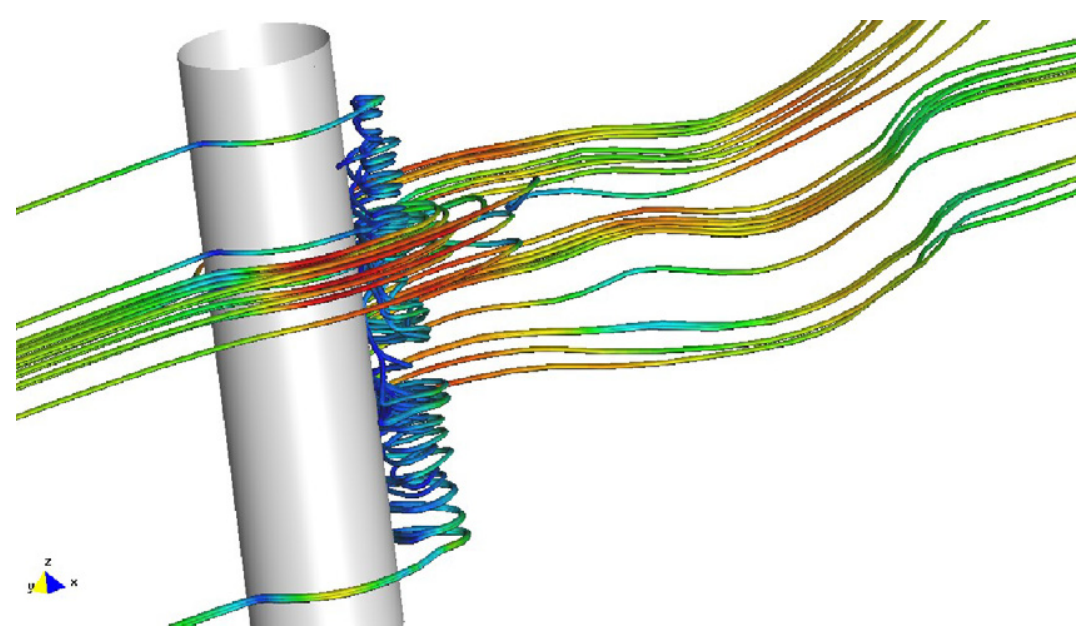

Fig. 17. Streamlines in the wake of the cylinder at $t=100 \mathrm{~s}$. 
91,316 nodes. The radial thickness of the layer of elements around the cylinder is 0.015 . The number of elements in the highly-refined inner mesh is 252,725 . The time step for the integration of Eqs. (34) is set to 0.05 $[45,46]$.

Fig. 16 shows the isosurface of the vorticity modulus $w$ for $w=0.3$ at $t=100 \mathrm{~s}$. Results clearly show the 3D character of the flow at that Reynolds number. Fig. 17 shows streamlines behind the cylinder at $t=100 \mathrm{~s}$, inside the recirculating area. It is clear the structure of the vortex created in the turbulent region. When the vortex gets enough energy then it detaches from the cylinder, generating the von Karman street vortexes. The Strouhal number computed from the shedding frequency yielded $S=0.2$. This value compares well with the experimental data shown in Fig. 14.

Similar good results for this problem for a higher Reynolds number are reported in [54].

\section{Conclusions}

The finite calculus (FIC) form of the fluid mechanics equations is a good starting point for deriving stabilized FEM for solving a variety of incompressible fluid flow problems. The matrix stabilization terms introduced by the FIC formulation here presented allow to obtain physically sound solutions in the presence of sharp gradients occuring for high Reynolds numbers without the need of introducing a turbulence model. Good numerical solutions have been obtained in the 2D and 3D examples solved with relatively coarse meshes for moderately high values of the Reynolds number. These results reinforce our conviction that the stabilization terms introduced by the FIC formulation suffice to provide good results for problems for which turbulence models are required using more classical numerical methods. The results also confirm the close link between the stabilized methods and turbulence models, which surely will be the object of much research in the near future.

\section{Acknowledgment}

This research was partially supported by project SEDUREC of the Consolider Programme of the Ministerio de Educación y Ciencia of Spain.

\section{References}

[1] O.C. Zienkiewicz, R.C. Taylor, P. Nietharasu, The finite element method, Fluid Mech., vol. 3, Elsevier, 2005.

[2] C. Hirsch, Numerical Computation of Internal and External Flows, Wiley, vol. 1, 1988, vol. 2, 1990.

[3] D.W. Kelly, S. Nakazawa, O.C. Zienkiewicz, A note on anisotropic balancing dissipation in the finite element method approximation to convective diffusion problems, Int. J. Num. Meth. Engrg. 15 (1980) 1705-1711.

[4] J.C. Heinrich, P.S. Hayakorn, O.C. Zienkiewicz, An upwind finite element scheme for two dimensional convective transport equations, Int. J. Num. Meth. Engrg. 11 (1977) 131-143.

[5] A.N. Brooks, T.J.R. Hughes, Streamline upwind Petrov-Galerkin formulation for convective dominated flows with particular emphasis on the incompressible Navier-Stokes equations, Comput. Meth. Appl. Mech. Engrg. 32 (1982) 199-259.

[6] T.J.R. Hughes, T.E. Tezduyar, Finite element methods for first-order hyperbolic systems with particular emphasis on the compressible Euler equations, Comput. Meth. Appl. Mech. Engrg. 45 (1984) 217-284.

[7] T.J.R. Hughes, M. Mallet, A new finite element formulations for computational fluid dynamics: III. The generalized streamline operator for multidimensional advective-diffusive systems, Comput. Meth. Appl. Mech. Engrg. 58 (1986) 305-328.

[8] T.J.R. Hughes, L.P. Franca, M. Balestra, A new finite element formulation for computational fluid dynamics. V Circumventing the Babuska-Brezzi condition: A stable Petrov-Galerkin formulation of the Stokes problem accommodating equal order interpolations, Comput. Meth. Appl. Mech. Engrg. 59 (1986) 85-99.

[9] L.P. Franca, S.L. Frey, Stabilized finite element methods: II. The incompressible Navier-Stokes equations, Comput. Meth. Appl. Mech. Engrg. 99 (1992) 209-233.

[10] T.E. Tezduyar, S. Mittal, S.E. Ray, R. Shih, Incompressible flow computations with stabilized bilinear and linear equal order interpolation velocity-pressure elements, Comput. Meth. Appl. Mech. Engrg. 95 (1992) 221-242.

[11] T.J.R. Hughes, G. Hauke, K. Jansen, Stabilized finite element methods in fluids: Inspirations, origins, status and recent developments, in: Robert L. Taylor, T.J.R. Hughes, E. Oñate, O.C. Zienkiewicz (Eds.), Recent Developments in Finite Element Analysis. International Center for Numerical Methods in Engineering (CIMNE), Barcelona, Spain, 1994, pp. $272-292$.

[12] M. Storti, N. Nigro, S.R. Idelsohn, Steady state incompressible flows using explicit schemes with an optimal local preconditioning, Comput. Meth. Appl. Mech. Engrg. 124 (1995) 231-252. 
[13] M.A. Cruchaga, E. Oñate, A finite element formulation for incompressible flow problems using a generalized streamline operator, Comput. Meth. Appl. Mech. Engrg. 143 (1997) 49-67.

[14] M.A. Cruchaga, E. Oñate, A generalized streamline finite element approach for the analysis of incompressible flow problems including moving surfaces, Comput. Meth. Appl. Mech. Engrg. 173 (1999) 241-255.

[15] T.J.R. Hughes, L.P. Franca, G.M. Hulbert, A new finite element formulation for computational fluid dynamics: VIII. The Galerkin/ least-squares method for advective-diffusive equations, Comput. Meth. Appl. Mech. Engrg 73 (1989) 173-189.

[16] J. Donea, A Taylor-Galerkin method for convective transport problems, Int. J. Num. Meth. Engrg. 20 (1984) $101-119$.

[17] R. Löhner, K. Morgan, O.C. Zienkiewicz, The solution of non-linear hyperbolic equation systems by the finite element method, Int. J. Num. Meth. Fluids 4 (1984) 1043.

[18] R. Codina, M. Vazquez, O.C. Zienkiewicz, A general algorithm for compressible and incompressible flow - Part III. The semi-implicit form, Int. J. Num. Meth. Fluids 27 (1998) 13-32.

[19] R. Codina, O.C. Zienkiewicz, CBS versus GLS stabilization of the incompressible Navier-Stokes equations and the role of the time step as stabilization parameter, Commun. Num. Meth. Engrg. 18 (2) (2002) 99-112.

[20] T.J.R. Hughes, Multiscale phenomena: Green functions subgrid scale models bubbles and the origins of stabilized methods, Comput. Meth. Appl. Mech. Engrg. 127 (1995) 387-401.

[21] F. Brezzi, L.P. Franca, T.J.R. Hughes, A. Russo, $b=\int g$, Comput. Meth. Appl. Mech. Engrg. 145 (1997) $329-339$.

[22] R. Codina, J. Blasco, Stabilized finite element method for the transient Navier-Stokes equations based on a pressure gradient operator, Comput. Meth. Appl. Mech. Engrg. 182 (2000) 277-301.

[23] R. Codina, Stabilized finite element approximation of transient incompressible flows using orthogonal subscales, Comput. Meth. Appl. Mech. Engrg. 191 (2002) 4295-4321.

[24] J. Donea, A. Huerta, Finite Element Method for Flow Problems, Wiley, 2003.

[25] L. Nottale, Relativity in All Its States, Hachette, 1998 (in French).

[26] E. Oñate, Derivation of stabilized equations for advective-diffusive transport and fluid flow problems, Comput. Meth. Appl. Mech. Engrg. 151 (1998) 233-267.

[27] E. Oñate, Possibilities of finite calculus in computational mechanics, Int. J. Num. Meth. Engrg. 60 (1) (2004) $255-281$.

[28] E. Oñate, J. García, S. Idelsohn, Computation of the stabilization parameter for the finite element solution of advective-diffusive problems, Int. J. Num. Meth. Fluids 25 (1997) 1385-1407.

[29] E. Oñate, J. García, S. Idelsohn, An alpha-adaptive approach for stabilized finite element solution of advective-diffusive problems with sharp gradients, in: P. Ladeveze, J.T. Oden (Eds.), New Advances in Adaptive Computational Methods in Mechanics, Elsevier, 1998.

[30] E. Oñate, M. Manzan, A general procedure for deriving stabilized space-time finite element methods for advective-diffusive problems, Int. J. Num. Meth. Fluids 31 (1999) 203-221.

[31] E. Oñate, Multiscale computational analysis in mechanics using finite calculus: an introduction”, Comput. Meth. Appl. Mech. Engrg. $192(28-30)$ (2003) 3043-3059.

[32] E. Oñate, S. Idelsohn, A mesh free finite point method for advective-diffusive transport and fluid flow problems, Comput. Mech. 21 (1998) 283-292.

[33] E. Oñate, F. Zárate, S.R. Idelsohn, Finite element formulation for convective-diffusive problems with sharp gradients using finite calculus, Comput. Meth. Appl. Mech. Engrg. 195 (2006) 1793-1825.

[34] E. Oñate, J. Miquel, G. Hauke, Stabilized formulation for the advection-diffusion-absorption equation using finite calculus and linear finite elements, Comput. Meth. Appl. Mech. Engrg. 195 (33-36) (2006) 3926-3946.

[35] E. Oñate, J. Miquel, F. Zárate, Stabilized solution of the multidimensional advection-diffusion-absorption equation using linear finite elements, Comput. Fluids 36 (1) (2007) 92-112.

[36] C.A. Felippa, E. Oñate, Nodally exact Ritz discretizations of 1D diffusion-absorption and Helmholtz equations by variational FIC and modified equation methods, Comput. Mech. 39 (2) (2007) 91-111.

[37] E. Oñate, A stabilized finite element method for incompressible viscous flows using a finite increment calculus formulation, Comput. Meth. Appl. Mech. Engrg. 182 (1-2) (2000) 355-370.

[38] E. Oñate, C. Sacco, S.R. Idelsohn, A finite point method for incompressible flow problems, Comput. Visual. Sci. 2 (2000) 67-75.

[39] E. Oñate, J. García, A finite element method for fluid-structure interaction with surface waves using a finite calculus formulation, Comput. Meth. Appl. Mech. Engrg. 191 (2001) 635-660.

[40] J. García, E. Oñate, An unstructured finite element solver for ship hydrodynamic problems, J. Appl. Mech. 70 (2003) 18-26.

[41] S.R. Idelsohn, E. Oñate, F. Del Pin, A lagrangian meshless finite element method applied to fluid-structure interaction problems, Comput. Struct. 81 (2003) 655-671.

[42] E. Oñate, S.R. Idelsohn, F. Del Pin, R. Aubry, The particle finite element method. An overview, Int. J. Comput. Meth. 1 (2) (2004) 267-307.

[43] S.R. Idelsohn, E. Oñate, F. Del Pin, The Particle Finite Element Method: a powerful tool to solve incompressible flows with freesurfaces and breaking waves, Int. J. Num. Meth. Engrg. 61 (2004) 964-989.

[44] E. Oñate, J. García, S.R. Idelsohn, Ship hydrodynamics', in: E. Stein, R. de Borst, T.J.R. Hughes (Eds.), Encyclopedia of Computational Mechanics, vol. 3, Wiley, 2004, pp. 579-607 (Chapter 18).

[45] E. Oñate, J. García, S.R. Idelsohn, F. Del Pin, FIC formulations for finite element analysis of incompressible flows Eulerian ALE and Lagrangian approaches, Comput. Meth. Appl. Mech. Engrg. 195 (23-24) (2006) 3001-3037.

[46] E. Oñate, A. Valls, J. García, FIC/FEM formulation with matrix stabilizing terms for incompressible flows at low and high Reynold's numbers, Comput. Mech. 38 (4-5) (2006) 440-455. 
[47] E. Oñate, J. Rojek, R.L. Taylor, O.C. Zienkiewicz, Finite calculus formulation for incompressible solids using linear triangles and tetrahedra, Int. J. Num. Meth. Engrg. 59 (2004) 1473-1500.

[48] E. Oñate, R.L. Taylor, O.C. Zienkiewicz, J. Rojek, A residual correction method based on finite calculus, Eng. Comput. 20 (5/6) (2003) 629-658.

[49] J. Rojek, E. Oñate, R.L. Taylor, CBS-based stabilization in explicit solid dynamics, Int. J. Num. Meth. Engrg. 66 (2006) $1547-1568$.

[50] T.E. Tezduyar, Y. Osawa, Finite element stabilization parameters computed from element matrices and vectors, Comput. Meth. Appl. Mech. Engrg. 190 (2000) 411-430.

[51] T.E. Tezduyar, Computation of moving boundaries and interfaces and stabilization parameters, Int. J. Num. Meth. Fluids 43 (2003) $555-575$

[52] Tdyn, A finite element code for fluid-dynamic analysis, COMPASS Ingeniería y Sistemas SA, www.compassis.com, 2007.

[53] V. Kalro, T. Tezduyar, Parallel 3D computation of unsteady flows around circular cylinders, Parallel Comput. 23 (1997) 1235-1248.

[54] E. Oñate, A. Valls, J. García, Computation of turbulent flows using a finite element-finite element formulation, Int. J. Num. Meth. Fluids (January) (2007). 